\title{
Glycaemic control improves fibrin network characteristics in type 2 diabetes - A purified fibrinogen model
}

\author{
Marlien Pieters', Namukolo Covic', Francois H. van derWesthuizen², Chandrasekaran Nagaswami ${ }^{3}$, Yelena Baras ${ }^{3}$, \\ Du Toit Loots', Johann C. Jerling', Dale Elgar ${ }^{4}$, Kathryn S. Edmondson 3 , Danie G. van Zyl5, Paul Rheeder ${ }^{6}$, \\ John W. Weisel ${ }^{3}$ \\ 'Department of Nutrition, North-West University, Potchefstroom, South Africa; ${ }^{2}$ School of Biochemistry, North-West University, \\ Potchefstroom, South Africa; ${ }^{3}$ Department of Cell and Developmental Biology, University of Pennsylvania, Philadelphia, Pennsylvania, USA; \\ ${ }^{4}$ School of Pharmacy, North-West University, Potchefstroom, South Africa; ${ }^{5}$ Department of Internal Medicine, University of Pretoria, Pretoria, \\ South Africa; ${ }^{6}$ Division of Clinical Epidemiology, University of Pretoria, Pretoria, South Africa
}

\begin{abstract}
Summary
Diabetic subjects have been shown to have altered fibrin network structures. One proposed mechanism for this is non-enzymatic glycation of fibrinogen due to high blood glucose. We investigated whether glycaemic control would result in altered fibrin network structures due to decreased fibrinogen glycation. Twenty uncontrolled type 2 diabetic subjects were treated with insulin in order to achieve glycaemic control. Twenty age- and body mass index (BMI)-matched non-diabetic subjects were included as a reference group. Purified fibrinogen, isolated from plasma samples was used for analysis. There was a significant decrease in fibrinogen glycation $(6.8 \mathrm{I}$ to $5.02 \mathrm{~mol}$ glucose $/ \mathrm{mol} \mathrm{fi-}$ brinogen) with a corresponding decrease in rate of lateral aggregation (5.86 to 4.62 ) and increased permeability ( 2.45 to $2.85 x$ $\left.10^{-8} \mathrm{~cm}^{2}\right)$ and lysis rate $(3.08$ to $3.27 \mu \mathrm{m} / \mathrm{min}$ ) in the diabetic subjects after glycaemic control.These variables correlated with
\end{abstract}

\section{Keywords}

Diabetes, fibrin network, fibrinogen glycation, glycaemic control, hyperglycaemia

\section{Introduction}

Diabetes is a powerful and independent risk factor for cardiovascular disease, with atherosclerosis among the most serious complications of type 2 diabetes (1). Since fibrin is deposited at atherosclerotic lesions, the structure of the deposited fibrin network has come under investigation as a possible contributing risk factor for increased prevalence of atherosclerosis-related cardiovascular events. Fibrin networks can vary in structure from tight markers of glycaemic control. Fibrin clots of non-diabetic subjects had a significantly higher ratio of inelastic to elastic deformation than the diabetic subjects $(0.10$ vs. 0.09$)$. Although there was no difference in median fiber diameter between diabetic and non-diabetic subjects, there was a small increase in the proportion of thicker fibers in the diabetic samples after glycaemic control. Results from SDS-PAGE indicated no detectable difference in factor XIIla-crosslinking of fibrin clots between uncontrolled and controlled diabetic samples. Diabetic subjects may have altered fibrin network formation kinetics which contributes to decreased pore size and lysis rate of fibrin clots. Achievement of glycaemic control and decreased fibrinogen glycation level improves permeability and lysis rates in a purified fibrinogen model.

Thromb Haemost 2008; 99: 691-700

networks formed with thin, highly branched fibers, to looser networks made up of larger fibers. It has been demonstrated that myocardial infarction at a young age is indeed associated with a proneness to the formation of tight and rigid fibrin gel structures $(2,3)$. Fibrin clots with thinner fibers, more branch points and smaller intrinsic pores are in general more dense and resistant to lysis, thereby contributing to thrombotic risk (4).

For this reason, fibrin networks of diabetic patients have received considerable attention as a possible contributor to in- 
creased cardiovascular disease risk. One of the most important plausible mechanisms for alterations to fibrin networks of diabetic patients is the non-enzymatic glycation of fibrinogen in the presence of uncontrolled blood glucose levels, which may influence the functionality of the fibrinogen. Researchers have investigated this issue by using either in vitro glycated fibrinogen (5-9) or collecting samples from diabetic subjects containing in vivo glycated fibrinogen (10-14). When using diabetic samples, a further distinction was made in the use of either plasma (11-14) or purified fibrinogen models $(10,12,15)$ Apart from the differing approaches used, a vast array of variables was also measured, including both structural and functional characteristics, in order to gain a better understanding of the effects of diabetes on fibrin networks.

Interpreting results from studies where fibrinogen was glycated in vitro should be done with caution, as there is a significant difference between fibrinogen glycation in vitro and in vivo, making extrapolation to in vivo situations difficult. While using samples from diabetic patients may be more physiological, it should be kept in mind that the diabetic state has many other components that could potentially influence fibrin network structure, apart from fibrinogen glycation, such as increased lipid levels (16), increased fibrinogen concentration $(17,18)$ and glycation of other plasma proteins. In general, clots formed from diabetic patients were found to be denser, with thinner fibers and were less porous than those formed from healthy individuals ( 10 , $11,16)$. Pieters et al. (14) found, however, no difference in network characteristics of diabetic patients compared to healthy individuals, despite a significant difference in the level of fibrinogen glycation, when using plasma samples. In this study population, however, both the diabetic and healthy individuals had similar and raised fibrinogen levels. The fibrin network characteristics also correlated with baseline fibrinogen concentration but not with any of the markers of glycaemic control. Similar correlation patterns were observed by Jörneskog et al. (16) indicating the importance of plasma constituents other than fibrinogen glycation on fibrin network structure.

The ideal approach to examine the role of fibrinogen glycation on fibrin network structures of diabetic patients would be to conduct an intervention trial specifically designed to investigate the effect of glycaemic control on fibrin network structures. Most of the above mentioned studies on diabetic subjects, however, were cross-sectional by design, making the establishment of causal relationships impossible. To date, only two intervention studies have been performed on this topic, one investigating type 1 diabetic patients (16) and the other investigating type 2 diabetic subjects, performed by our group (14). The scarcity of studies such as these is not surprising since they require the unique combination of an intervention trial with patients and difficult and time-consuming structural and biophysical studies of clot networks. Two papers have been published from the study done by our group. The first describes the effect of glycaemic control on fibrinogen glycation and the association between fibrinogen glycation, fasting glucose and $\mathrm{HbAlc}$ (19). The second describes the effect of glycaemic control on fibrin network structure using plasma samples in order to include the possible contribution of individual fibrinogen concentrations on the fibrin networks (14). In order to determine the specific effects of fibrinogen glycation on fibrin network structures in the absence of other possibly confounding plasma proteins, we excluded the effects of other plasma components for the purpose of this paper, by purifying fibrinogen from the collected plasma samples, thereby establishing a purified fibrinogen model. The discussion will include interpretation of the results obtained from both plasma and purified fibrinogen models in order to demonstrate how results may be influenced by the model used.

\section{Materials and methods}

\section{Study design}

A minimum of 16 volunteers had to be included per group in order to achieve a difference of one standard deviation at $80 \%$ power and 5\% level of significance. To accommodate possible non-compliers and drop-outs, 20 volunteers were recruited per group. A parallel, controlled intervention with blinded assessment was used. Twenty black African type 2 diabetic subjects, uncontrolled on oral hypoglycaemic agents, were included and treated additionally with insulin until glycaemic control was achieved ( 4 out of 5 subsequent readings within normal glucose range). Following this initial period of improving and finally achieving glycaemic control, the patients then had to remain controlled for eight days before end blood samples were drawn. This eight day period was chosen in order to provide enough time for unglycated fibrinogen to be produced (half-life 3-4 days) after glycaemic control had been achieved. Twenty non-diabetic black African subjects were included as a reference group in order to control for variation over time. All subjects signed informed consent and ethical approval was obtained form the ethics committees of both the University of Pretoria and the North-West University, South Africa.

\section{Subjects \\ Type 2 diabetic subjects}

Inclusion criteria: patients had to be uncontrolled $(\mathrm{HbA1C}>$ 9\%) on maximum dose combination oral hypoglycaemic medication (Metformin and Sulphonylureas); body mass index (BMI) $>25 \mathrm{~kg} / \mathrm{m}^{2} ; 40-65$ years of age with blood pressure sufficiently controlled $(<140 / 90 \mathrm{mmHg})$ not to necessitate treatment change during intervention (hidrochlorothiazide, ACE-inhibitor - Perindopril and Calcium antagonist - Nifedipine).

Exclusion criteria: major surgery in the preceding six months; macrovascular complications; diseases that may influence haemostasis (e.g. thrombocytopenia, cancer, liver disease); patients on aspirin, warfarin, steroids, hormone replacement therapy or non-steroidal anti-inflammatory drugs; proteinuria on urine dipstick (>300 mg/day) or acute infection.

\section{Non-diabetic controls}

Non-diabetic subjects with matching anti-hypertensive druguse, age, gender and BMI were recruited. The same inclusion and exclusion criteria as for the type 2 diabetic subjects were adhered to. Baseline oral glucose tolerance tests were done to rule out diabetes.

\section{Study protocol}

The intervention in the diabetic group consisted of three phases. 


\section{Phase I}

On the first visit, diabetic subjects were taught how to do selfglucose monitoring, co-ordination of insulin use with meals, symptoms and management of hypoglycaemic events and the use of glucagon. Fasting capillary glucose was measured daily for one week.

\section{Phase 2}

Subjects received 10 IU basal analogue insulin Glargine (Lantus, Sanofi-Aventis Pharmaceuticals, Paris, France) daily at 10 p.m. in addition to current treatment of maximum dose oral hypoglycaemic treatment. Metformin use was unchanged from before and during the intervention. Insulin administration was adjusted individually until four out of five subsequent fasting values were less than $7.2 \mathrm{mM}$. Sulphonylureas were stopped.

\section{Phase 3}

Post-prandial glucose was now controlled with pre-meal administration of short-acting insulin Aspart (Novo Nordisk, Bagsværd, Denmark) as required to achieve post-prandial glucose values of less than $10 \mathrm{mM}$. Once both fasting and post-prandial glycaemic control was achieved, the subjects remained on treatment for eight days. Baseline blood samples and anthropometric measurements were collected at the end of phase 1 and end samples and measurements at the end of phase 3. Blood samples of non-diabetic subjects were drawn within one week of blood sampling from their matched diabetic subjects.

\section{Blood sampling}

Fasting venous blood samples were drawn with minimal stasis by a physician before 10 a.m. Blood for venous glucose determination, was collected into sodium fluoride tubes. EDTA blood was collected for the determination of HbA1C. Citrate blood (60 $\mathrm{ml}$ ) was collected for the determination of fibrinogen concentration, fibrinogen glycation and for fibrinogen purification. Blood was centrifuged for 15 minutes (min) at $2,000 \mathrm{~g}$ at $4^{\circ} \mathrm{C}$ within $30 \mathrm{~min}$ of collection. Serum and plasma were stored at $-82^{\circ} \mathrm{C}$ until analysis. Baseline and end samples of an individual were analyzed in the same run for all experiments. Scanning electron microscopy (SEM), and viscoelastic measurements were performed in a different laboratory than the other analyses.

\section{Glucose and $\mathrm{HbAIC}$}

Plasma glucose and baseline $\mathrm{HbA1C}$ were measured on a Synchron LX clinical System (Beckman Coulter Inc., Fullerton, CA, USA).

\section{Fibrinogen concentration, purification and glycation}

Plasma fibrinogen (modified Clauss method) was measured on an Automated Coagulation Laboratory 200 (Instrumentation Laboratories, Milan, Italy) (between-run $\mathrm{CV}=3 \%$ ). Fibrinogen was purified from the plasma of each subject using IF-1(fibrinogen monoclonal antibody, Kamiya Biomedical Company, Seattle, WA, USA) affinity chromatography as described previously (20). Purified fibrinogen was run on $10 \%$ SDS PAGE gels to confirm purity and the absence of degradation of the fibrinogen preparations. No bands, apart from the three intact fibrinogen chains were present on the gels. The broad-range mo- lecular-weight standard used ranged from 35 to $250 \mathrm{kD}$. Fibrinogen glycation was measured in duplicate, with a two-reagent enzymatic assay (GlyPro ${ }^{\circledR}$ assay, Genzyme Diagnostics, Cambridge, MA, USA; between-run $\mathrm{CV}=5 \%$ ) as described previously (14).

\section{Clot permeability}

Permeability was measured essentially as described previously $(11,21)$. Purified fibrinogen $(1 \mathrm{mg} / \mathrm{ml})$ clots of subjects were prepared in triplicate in $3 \mathrm{~cm}$ sections of $1 \mathrm{ml}$ pipette tips. Fibrinogen was clotted with the addition of $1 \mathrm{U} / \mathrm{ml}$ bovine thrombin, $5 \mathrm{mM} \mathrm{CaCl}_{2}$ and incubated for $60 \mathrm{~min}$ at $25^{\circ} \mathrm{C}$ in a moist atmosphere. Buffer was permeated through at a pressure height of $6 \mathrm{~cm}$ $(\mathrm{CV}=7 \%)$. These conditions were selected in order to prevent clots from collapsing. Permeability (Ks), an indication of the intrinsic pore size of the network, was then calculated (22).

\section{Turbidity measurements}

Turbidity experiments were performed in duplicate with purified fibrinogen $(1 \mathrm{mg} / \mathrm{ml}), 1 \mathrm{U} / \mathrm{ml}$ bovine thrombin and $5 \mathrm{mM} \mathrm{CaCl}_{2}$ (final concentrations). Absorbance was measured at $405 \mathrm{~nm}$ every 7 seconds (s) for the first 2 min after addition of thrombin and $\mathrm{CaCl}_{2}$, then every $15 \mathrm{~s}$ for the next $30 \mathrm{~min}$ and then every 5 min up to a total time of 60 min on a Multiscan Ascent spectrophotometer (Labsystems, Helsinki, Finland). Lag phase, slope and maximum absorbance were recorded. The lag phase represents the time required for fibrin fibers to grow sufficiently to allow lateral aggregation and was taken at the point where absorbance increased 0.015 from baseline. The slope, calculated at half maximum absorbance, represents the rate of increase of turbidity, and the maximum absorbance, calculated as the absorbance after $60 \mathrm{~min}$ minus that at baseline, is an indication of average fiber size.

In order to determine whether fibrinogen glycation is one of the mechanisms of action through which fibrin network architecture is altered in diabetes, the experiments mentioned below were performed on subjects with striking differences in the degree of fibrinogen glycation. Due to the difficulty and time consuming nature of the experiments, a subset of 12 subjects consisting of seven diabetic subjects who showed the largest decrease in fibrinogen glycation from baseline to end and five randomly selected non-diabetic subjects were investigated for this purpose.

\section{SEM of fibrin clots and fiber diameter measurement}

SEM was used to determine the fibrin structure of clots formed from purified fibrinogen of diabetic and control subjects. Clots were formed by addition of $0.5 \mathrm{U} / \mathrm{ml} \alpha$-human thrombin and $3 \mathrm{mM} \mathrm{CaCl}_{2}$ to $1 \mathrm{mg} / \mathrm{ml}$ fibrinogen in $0.15 \mathrm{M} \mathrm{NaCl}, 0.05 \mathrm{M}$ Tris$\mathrm{HCl}, \mathrm{pH}$ 7.4. Samples were prepared as described previously (23). Clots were observed and photographed digitally in many different areas, using a scanning electron microscope (XL 20, FEI, Hillsboro, OR, USA). Fiber diameters were measured from micrographs at 10,000 x magnification using ImageJ software (National Institutes of Health, USA). The thicknesses of at least 100 different fibers were measured per micrograph, with at least six micrographs imaged for each patient. 


\section{Viscoelastic measurements of fibrin clots}

A Plazek torsion pendulum was used to determine visco-elastic properties of clots, including clot stiffness (24), in triplicate, formed from purified fibrinogen from diabetic subjects and controls under the same conditions as described above. Data recorded was used to calculate G' (the dynamic storage modulus), $\mathrm{G}^{\prime \prime}$ (the loss modulus) and the $\tan \delta$ (loss tangent), which is a function of $G^{\prime \prime}$ over $G^{\prime}$ and gives information about irreversible deformation of the clot (24).

\section{Lysis rate of fibrin clots measured by confocal microscopy}

Fluorescein isothiocyanate (FITC) (Pierce, Rockford, IL, USA) labeled fibrin clots were prepared using $1 \mathrm{mg} / \mathrm{ml}$ purified fibrinogen, $0.15 \mathrm{mg} / \mathrm{ml}$ FITC-labeled fibrinogen, $1 \mathrm{U} / \mathrm{ml}$ bovine thrombin and $5 \mathrm{mM} \mathrm{CaCl}_{2}$. The fibrinogen was labeled with FITC as described by Sakharov et al. (25). The clots were prepared in micro-chambers and incubated for $45 \mathrm{~min}$ at $37^{\circ} \mathrm{C}$ in a moist atmosphere before being lysed with a lysis buffer containing $1 \mu \mathrm{g} /$ $\mathrm{ml}$ tissue plasminogen activator (tPA) (Actilyse from Boehringer Ingelheim, Ingelheim, Germany), $1 \mathrm{mg} / \mathrm{ml}$ bovine serum albumin (BSA) (Sigma-Aldrich, St Louis, USA), $210 \mu \mathrm{g} / \mathrm{ml}$ human gluplasminogen (American Diagnostica, Stamford, CT, USA), 140 $\mathrm{mM} \mathrm{NaCl}$ and $50 \mathrm{mM}$ Tris, $\mathrm{pH}$ 7.4. After a $15 \mathrm{~min}$ incubation at $37^{\circ} \mathrm{C}$ in a moist atmosphere, lysis rate was measured by following the lysis front on a PCM 2000 Nikon confocal laser scanning microscope linked to a Nikon TE300 inverted microscope equipped with an ApoPlanar 1.4NA $60 \mathrm{x}$ oil immersion objective. A 5-W argon ion laser was used in combination with a $488 \mathrm{~nm}$ band-pass filter for excitation. Emission was monitored at $505 \mathrm{~nm}$. A pinhole of 5 micron and a $10 \%$ neutral density filter were used to limit bleaching of the FITC-label. Images were obtained using a standardized format: $107 \times 107 \times 16 \mu \mathrm{m}$ with four optical sections collected at z-intervals of $4 \mu \mathrm{m}$. Lysis front velocity was determined at three different sites along the lysis front in triplicate, in order to calculate the mean lysis rate in $\mu \mathrm{m} / \mathrm{min}$.

\section{Analysis of fibrin cross-linking by SDS-PAGE}

Initial SDS-PAGE results indicated that the fibrin clots made from the purified fibrinogen contained very little if any factor (F)XIII. In order to determine whether fibrinogen glycation has an effect on FXIIIa crosslinking, we performed SDS-PAGE analysis with and without added human FXIII (American Diagnostica) using an incubation time series. FXIII $(22 \mu \mathrm{g} / \mathrm{ml})$ was added to the purified fibrinogen $(1 \mathrm{mg} / \mathrm{ml})$ and clots were form- ed by the addition of $1 \mathrm{U} / \mathrm{ml}$ bovine thrombin and $5 \mathrm{mM} \mathrm{CaCl}_{2}$ (final concentrations) at $21^{\circ} \mathrm{C}$ in $20 \mu \mathrm{l}$ reaction mixtures which were then incubated for 10, 45 or $90 \mathrm{~min}$. Control samples without added FXIII were incubated for $90 \mathrm{~min}$, in order to demonstrate the absence of FXIII in the purified fibrinogen. The ligating reaction was stopped by solubilization in $6 \mathrm{M}$ urea, $40 \mathrm{mM}$ dithiothreitol and $2 \%(\mathrm{w} / \mathrm{v}) \mathrm{SDS}$ at $37^{\circ} \mathrm{C}$ for $45 \mathrm{~min}$. Gel electrophoresis $[8 \%(\mathrm{w} / \mathrm{v})$ acrylamide] was performed by the procedure of Laemmli (26). Samples of $6 \mu \mathrm{g}$ protein per lane were analyzed and a broad-range molecular weight standard (Amersham) was used as a reference. Coomassie brilliant blue R (Biorad, Hercules, CA, USA) was used for staining. The gel was photographed on a SynGene bio-imaging system and peaks were identified and quantified using GeneTools software version 3.06 (SynGene, Cambridge, UK ).

\section{Statistical methods}

The computer software package, Statistica (Statsoft Inc., Tulsa, OK, USA) was used for statistical analysis. Data is presented as median $\left[25^{\text {th }} ; 75^{\text {th }}\right.$ percentile]. Differences in baseline characteristics as well as differences in changes during the intervention between the two groups were determined using the Mann-Whitney U test. Differences from baseline to end within each group were determined using the Wilcoxon Matched Pairs test. Once statistical significance has been indicated, effect sizes can be used to determine whether this significance is likely to be important in practice. The effect size is independent of sample size and is an objective measure of the likelihood of a difference having a practical/clinical significance. Effect sizes were calculated according to the following formula: $\mathrm{r}=\mathrm{z} / \sqrt{n}_{\mathrm{n}}$ for non-parametric data ( $\mathrm{z}$ is the $\mathrm{z}$-statistic obtained from the non parametric test and $\mathrm{n}$ is the number of observations). The likelihood of the found statistical significance being practically relevant, is reported as effect size (r) and can be interpreted as follows; $r=0.1$ is a small likelihood, $r=0.3$ is a medium likelihood and $r=0.5$ is a large likelihood for non-parametric data (27). Spearman-correlation was used for determining correlation coefficients.

\section{Results}

Two of the non-diabetic subjects failed to return for end blood sampling and were hence excluded from the study. A summary of the most relevant baseline characteristics are presented in Table 1 while the detailed baseline characteristics of the study population have been presented in detail elsewhere (14).

\begin{tabular}{|c|c|c|}
\hline Variables & Type 2 diabetic subjects & Non-diabetic subjects \\
\hline $\begin{array}{l}\text { Patients (n) } \\
\text { Sex (male/female) } \\
\text { Age (years)\# } \\
\text { HbAlc (\%) } \\
\text { Venous glucose (mM)\# } \\
\text { Fasting Insulin (mU/l) } \\
\text { Insulin resistance (HOMA)\# } \\
\text { Fibrinogen (g/l) \# } \\
\text { Duration of diabetes (years) }\end{array}$ & $\begin{array}{l}20 \\
6 / 14 \\
53.0(49.1 ; 56.9) \\
\text { II.7 }[9.50 ; 13.8]^{\mathrm{a}} \\
14.6(10.8 ; 18.4)^{\mathrm{a}} \\
\text { II.0[6.70; 15.8] } \\
5.18(3.99 ; 6.95)^{\mathrm{b}} \\
4.25(3.88 ; 4.63) \\
\text { II.0 }(8.00 ; 15.0)\end{array}$ & $\begin{array}{l}18 \\
7 /|| \\
52.9(49.2 ; 56.6) \\
5.60[5.30 ; 5.90]^{\mathrm{a}} \\
5.18(4.56 ; 5.80)^{\mathrm{a}} \\
\mid 3.7[8.9 ; 28.8] \\
3.1 \mid(2.29 ; 7.42)^{\mathrm{b}} \\
4.02(3.59 ; 4.45)\end{array}$ \\
\hline
\end{tabular}

Table I: Baseline characteristics of subjects. Adapted from (14). Note: ${ }^{\text {a }} \mathrm{P}<0.00$ I; b $\mathrm{P}<0.05$; \# Data normally distributed and reported as mean ( $95 \%$ confidence interval); Insulin resistance $=($ fasting insulin $\times$ fasting venous glucose)/22.5. 


\begin{tabular}{|c|c|c|c|c|c|c|c|c|c|c|c|c|c|c|c|c|c|}
\hline 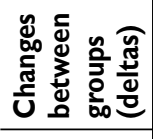 & 2 & 产 & $\frac{0}{0}$ & 능 & స్రి & $\begin{array}{l}0 \\
0\end{array}$ & & ठั & 㞼 & 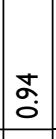 & $\frac{20}{0}$ & $\bar{m}$ & f̊. & fo & $\begin{array}{l}0 \\
0 \\
0\end{array}$ & $\begin{array}{c}\infty \\
0 \\
0\end{array}$ & $\stackrel{\infty}{\infty} \underset{0}{\circ}$ \\
\hline \multirow{4}{*}{ 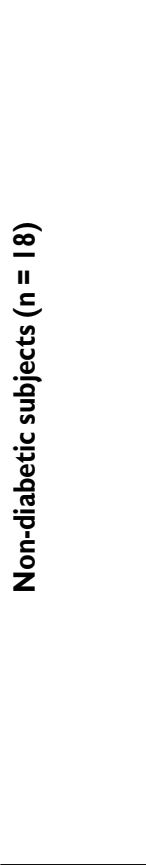 } & a & ֻ̊. & $\hat{o}$ & 息 & \begin{tabular}{|l|}
0 \\
0 \\
0
\end{tabular} & $\begin{array}{l}0 \\
\stackrel{2}{0} \\
\end{array}$ & & $\begin{array}{l}\text { o. } \\
\text { o. }\end{array}$ & \begin{tabular}{|c} 
o. \\
0 \\
0
\end{tabular} & $\begin{array}{l}\infty \\
0 \\
0\end{array}$ & $\begin{array}{l}\infty \\
0 \\
0 \\
0\end{array}$ & $\begin{array}{l}0 \\
0 \\
0 \\
0\end{array}$ & $\begin{array}{c}\stackrel{\leftrightarrow}{\tilde{O}} \\
0\end{array}$ & 号 & $\begin{array}{l}0 \\
0 \\
0\end{array}$ & 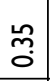 & $\begin{array}{l}\text { oे } \\
0 \\
0\end{array}$ \\
\hline & 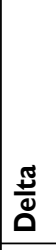 & 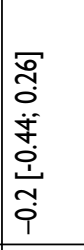 & 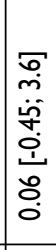 & 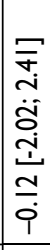 & 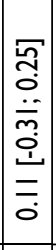 & 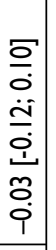 & 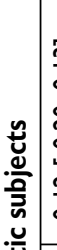 & 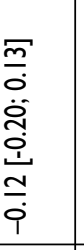 & 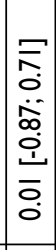 & 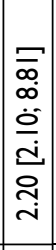 & 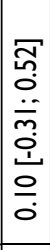 & 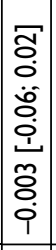 & 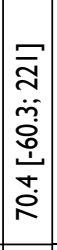 & 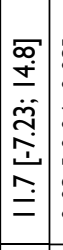 & $\begin{array}{l}\overline{0} \\
0 \\
0 \\
\dot{0} \\
0 \\
\dot{1} \\
\tilde{0} \\
0\end{array}$ & 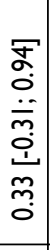 & 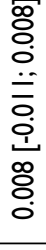 \\
\hline & \begin{tabular}{|l}
$\mathbf{z}$ \\
\end{tabular} & 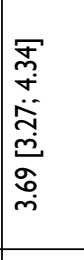 & 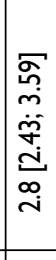 & 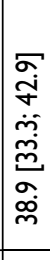 & 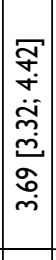 & 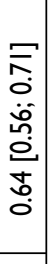 & 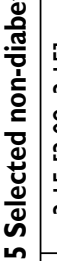 & 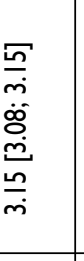 & 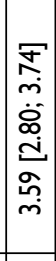 & 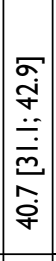 & 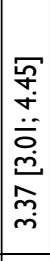 & 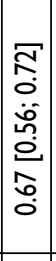 & 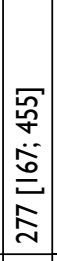 & 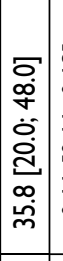 & \begin{tabular}{l|}
$\frac{m}{0}$ \\
\\
$\overline{\overline{0}}$ \\
$\overline{\overline{0}}$ \\
$\overline{\overline{0}}$
\end{tabular} & 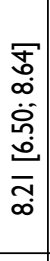 & 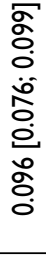 \\
\hline & 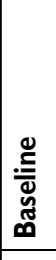 & 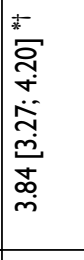 & 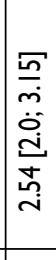 & $\begin{array}{l}\sigma \\
\sigma \\
\dot{j} \\
\dot{0} \\
\dot{0} \\
0 \\
0 \\
\infty \\
\infty\end{array}$ & 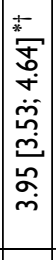 & \begin{tabular}{l}
\multicolumn{2}{c}{} \\
0 \\
0 \\
\\
0 \\
0 \\
0 \\
0 \\
0
\end{tabular} & & 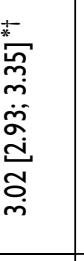 & 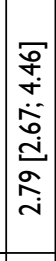 & 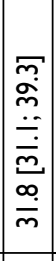 & 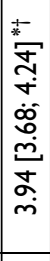 & 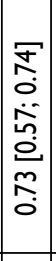 & 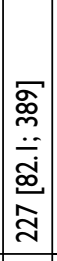 & 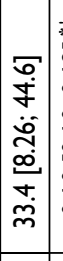 & 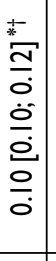 & 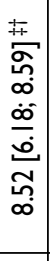 & 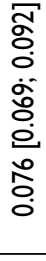 \\
\hline \multirow{4}{*}{ 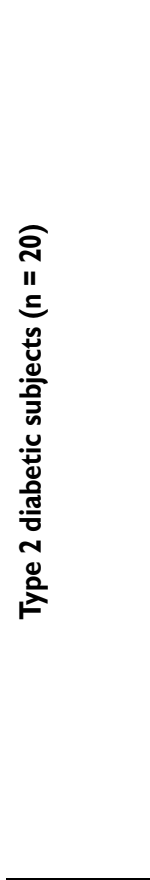 } & a & $\begin{array}{l}+ \\
\dot{O} \\
\dot{Q} \\
\dot{v}\end{array}$ & 官 & 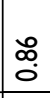 & $\overline{\tilde{\sigma}}$ & $\frac{\sigma}{0}$ & & ס̊ & $\begin{array}{l}\text { to } \\
0\end{array}$ & 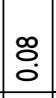 & 蒿 & $\begin{array}{l}\infty \\
0 \\
0\end{array}$ & \begin{tabular}{|c}
$\stackrel{2}{o}$ \\
$\tilde{O}$
\end{tabular} & $\frac{\infty}{0}$ & శ̊ & $\begin{array}{l}\tau \\
0 \\
0\end{array}$ & 웅 \\
\hline & $\frac{\text { I }}{\frac{\pi}{0}}$ & 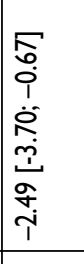 & 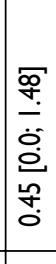 & 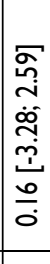 & 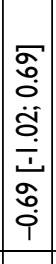 & 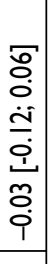 & 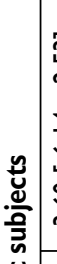 & 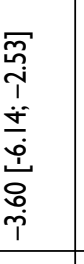 & 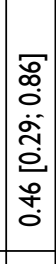 & 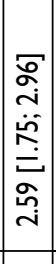 & 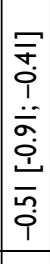 & 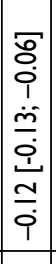 & 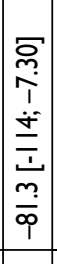 & 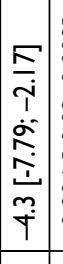 & $\begin{array}{l}\sigma \\
\delta \\
0 \\
\dot{0} \\
\dot{0} \\
\dot{1} \\
\stackrel{0}{0} \\
0\end{array}$ & 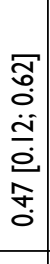 & 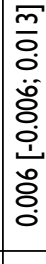 \\
\hline & 悹 & 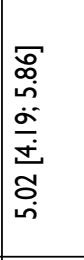 & 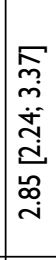 & 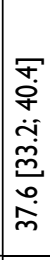 & 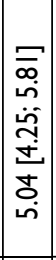 & 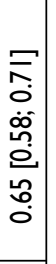 & 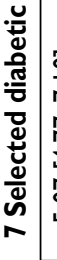 & 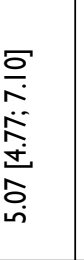 & 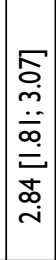 & 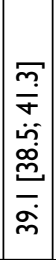 & 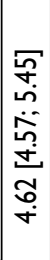 & 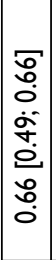 & 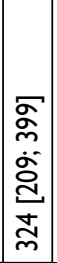 & 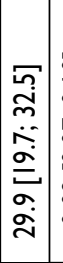 & $\begin{array}{l}\text { 응 } \\
0 \\
\ddot{0} \\
0 \\
0 \\
0 \\
0\end{array}$ & 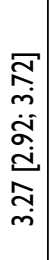 & 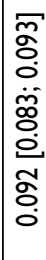 \\
\hline & 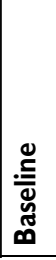 & 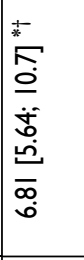 & 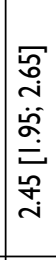 & 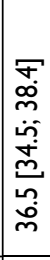 & 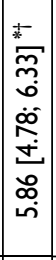 & $\begin{array}{l}\infty \\
0 \\
0 \\
\dot{0} \\
0 \\
0 \\
\hat{0} \\
0 \\
0\end{array}$ & & 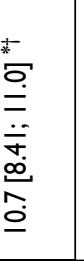 & 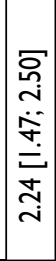 & 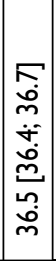 & 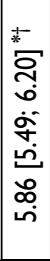 & 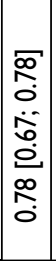 & 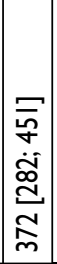 & 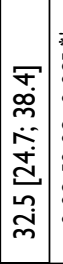 & 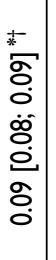 & 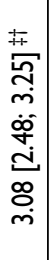 & 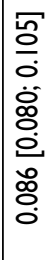 \\
\hline $\begin{array}{l}\frac{0}{0} \\
\frac{\pi}{\frac{\pi}{5}} \\
\text { ग }\end{array}$ & & 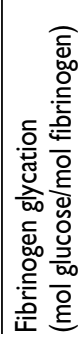 & 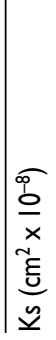 & 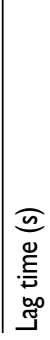 & $\left|\begin{array}{c}\frac{0}{0} \\
\text { o }\end{array}\right|$ & 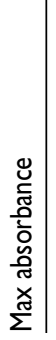 & & 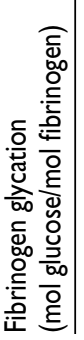 & $\left|\begin{array}{l}\tilde{p} \\
\underline{0} \\
x \\
\tilde{E} \\
\underline{E} \\
\underline{y}\end{array}\right|$ & 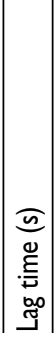 & & 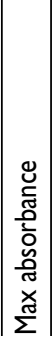 & 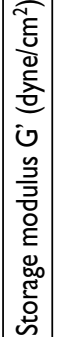 & 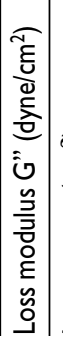 & 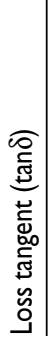 & 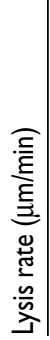 & 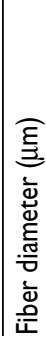 \\
\hline
\end{tabular}




\section{Fibrinogen glycation}

There was a significant decrease in the level of fibrinogen glycation upon achievement of glycaemic control (6.81 vs. $5.02 \mathrm{~mol}$ glucose/mol fibrinogen) (Table 2) as well as a significantly higher level of fibrinogen glycation in the diabetic compared to the non-diabetic subjects $(6.81$ vs. $3.84 \mathrm{~mol}$ glucose/mol fibrinogen) at baseline. For the sub-sample, selected based on the largest decrease of fibrinogen glycation during the intervention, the level of glycation decreased from 10.7 to $5.07 \mathrm{~mol}$ glucose $/ \mathrm{mol}$ fibrinogen. Effect size calculations indicated that these differences are large enough to not only have statistical significance but also have possible practical relevance.

\section{Clot permeability}

Clot permeability reflects the clot structure, specifically the average pore size. The results indicated both a statistically as well as a large likelihood of a practically relevant increase in permeability from baseline to end in the total diabetic group ( 2.45 to $\left.2.85 \times 10^{-8} \mathrm{~cm}^{2}\right)$. This relatively small but consistent increase was also observed in the sub-sample (2.24 to $\left.2.84 \times 10^{-8} \mathrm{~cm}^{2}\right)$ (Table 2 ). There was no change in permeability in the non-diabetic subjects, nor was there any difference between the diabetic and nondiabetic subjects at baseline. There was additionally a significant correlation between permeability and $\mathrm{HbAlc}$ at baseline $(r=-0.63 ; p=0.038)$.

\section{Turbidity measurements}

Turbidity curves are used to characterize the kinetics of polymerization and clot structure. No difference in the lag time (time for fibrinopeptide cleavage and formation of oligomers) or maximum absorbance (average cross-sectional area of fibers) was observed between the uncontrolled diabetic, controlled diabetic or the non-diabetic subjects (Table 2). The slope of increase in turbidity (rate of lateral aggregation) for the diabetic subjects was, however, significantly higher than that of the non-diabetic subjects at baseline, both for the total group (5.86 vs. 3.95) as well as for the sub-sample (5.86 vs. 3.94). The slope tended to decrease in the total diabetic group, and in the sub-sample the decrease reached significance with achievement of glycaemic control (5.86 to 4.62). This decrease was consistent for each subject compared to the normal variation observed in the non-diabetic group. These effects also had a large likelihood of practical relevance. Slope correlated significantly with level of fibrinogen glycation $(\mathrm{r}=0.65, \mathrm{p}=0.04), \mathrm{HbA1c}(\mathrm{r}=0.71, \mathrm{p}=0.02)$, venous glucose $(\mathrm{r}=0.77, \mathrm{p}=0.009)$ and negatively with lysis rate $(\mathrm{r}=-0.77, \mathrm{r}=0.009)$.

\section{Fiber diameter}

Clot structure was observed directly by SEM. The median fiber diameter of the clots from diabetic and non-diabetic subjects was similar (Table 2 and Fig. 1) at baseline and no significant change in the median fiber diameter was observed after achieving glycaemic control (Table 2). There was, however, a small increase in the proportion of thicker fibers in the clots of the diabetic subjects after glycaemic control as measured from the SEM micrographs, indicating a less homogeneous clot structure (Fig. 2).

\section{Viscoelastic properties}

The mechanical properties of the clots are important for their functions. There was no significant difference in dynamic storage modulus (elastic properties) or loss modulus (viscous properties) between the uncontrolled diabetic, controlled diabetic or the non-diabetic subjects (Table 2). The results showed, however a statistically significant difference in the loss tangent $(\tan \delta)$ measurement (ratio of inelastic to elastic component) between the diabetic and non-diabetic subjects ( 0.09 vs. 0.10$)$ at baseline, reflecting a lower proportion of the inelastic component in the fibrin clots of diabetic patients.

\section{Lysis rate}

Fibrinolysis rates were determined by measurement of the lysis front velocity using confocal microscopy after addition of tPA to

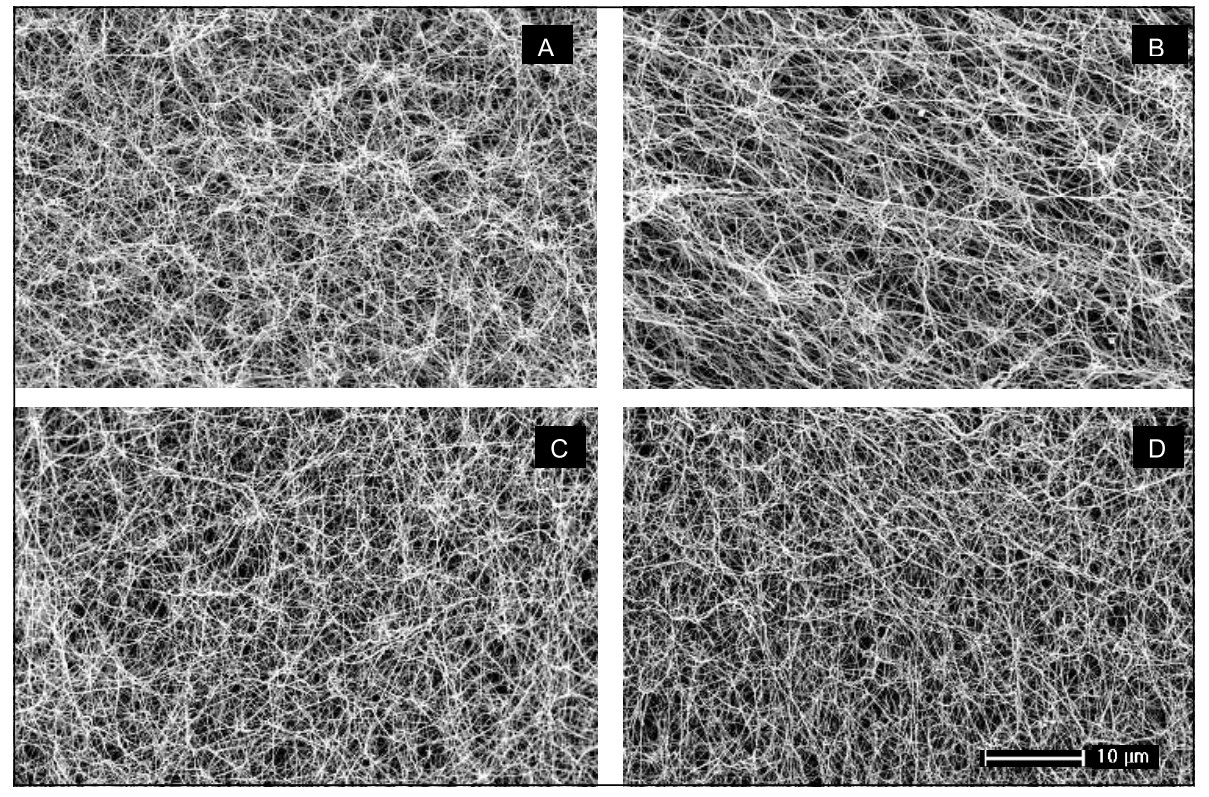

Figure I: Representative scanning electron micrographs of fibrin clots. A) Nondiabetic subject at baseline; B) Non-diabetic subject at end measurement; C) Uncontrolled diabetic subject; D) Controlled diabetic subject. 


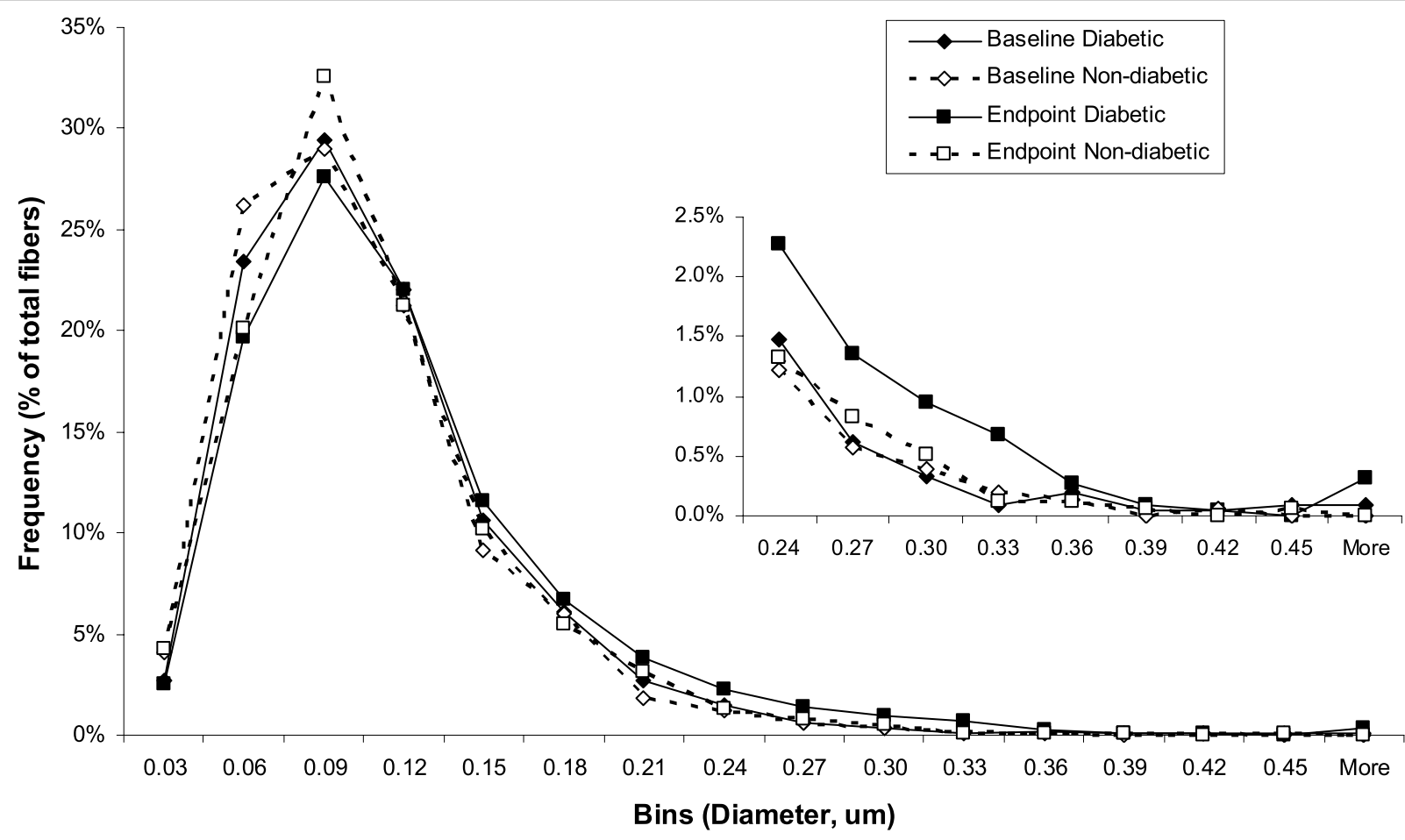

Figure 2: Histogram of fiber diameter distribution in the subsample of diabetic and non-diabetic subjects as calculated from SEM images.

the edge of a clot (Fig. 3). The diabetic subjects had a lower lysis rate than the non-diabetic subjects ( $3.08 \mathrm{vs} .8 .52 \mu \mathrm{m} / \mathrm{min})$. While the statistical significance was borderline $(\mathrm{p}=0.06)$, the likelihood of a practical relevance was considered to be large $(\mathrm{r}=0.54)$ (Table 2). A significant increase in lysis rate was observed with achievement of glycaemic control in the diabetic subjects (3.08 to $3.27 \mu \mathrm{m} / \mathrm{min}$ ). Although this increase was small in comparison to the difference between the diabetic and non-diabetic subjects, it was seen consistently in each diabetic subject measured. Lysis rate showed significant negative correlations with the level of fibrinogen glycation $(\mathrm{r}=-0.63, \mathrm{p}=0.028), \mathrm{HbA1c}(\mathrm{r}=-0.64$, $\mathrm{p}=0.026)$, fasting venous glucose $(\mathrm{r}=-0.60, \mathrm{p}=0.039)$ and slope $(\mathrm{r}=-0.77, \mathrm{p}=0.009)$.

\section{Effect of fibrinogen glycation on FXIIla cross-linking}

The fibrinogen isolated from subject plasma, contained very little if any FXIII (Fig. 4; lanes 7 and 8), indicating that the foregoing experiments were performed with non-crosslinked fibrin clots. When clots were crosslinked by the addition of FXIII, no difference in $\alpha$-chain disappearance rate could be observed between the non-diabetic, uncontrolled diabetic, and controlled diabetic subjects, as there appeared to be no significant difference in the percentage $\alpha$-chain density at any of the three time points (10 min: $19.7 \pm 6.0 ; 22.6 \pm 1.7 ; 21.6 \pm 3.9 ; 45 \mathrm{~min}: 6.6 \pm$ $4.6 ; 9.7 \pm 3.3 ; 12.4 \pm 3.6$; and $90 \mathrm{~min}: 2.6 \pm 3.6 ; 3.5 \pm 2.0 ; 6.2 \pm$ 4.1 of incubation with FXIIIa) using SDS-PAGE. Differences in $\gamma$-chain disappearance could not be measured as $\gamma$-chain crosslinking was already completed in all the subjects by $10 \mathrm{~min}$ (Fig. 4; lanes 1 and 2).

\section{Discussion}

This is the first intervention trial investigating the effect of glycaemic control on fibrin network structures of type 2 diabetic subjects using isolated fibrinogen. The network structures obtained from plasma clots from this same study, were reported previously (14). Using the plasma model, no difference in porosity, compaction and kinetics of clot formation between the diabetic and non-diabetic subjects at baseline was observed. Nor were any changes observed during the intervention in these variables in the diabetic group, despite a significant reduction in the level of fibrinogen glycation (14). This observed lack of effect could in part be explained by the fibrinogen concentration of the subjects. Both the non-diabetic and diabetic subjects had similar and raised fibrinogen concentrations and the results showed that the fibrin network characteristics measured were related to fibrinogen concentration and not to any of the glucose control markers. Fibrinogen concentration is acknowledged as one of the most important kinetic factors involved in the formation of the fibrin network and its final structure (28).

As described in this study, a purified fibrinogen model enables us to determine the effects of fibrinogen glycation on fibrin network structure independent of other plasma components. In contrast to that reported using plasma, there was a significant increase in permeability with achievement of glycaemic control and consequent decrease in the level of fibrinogen glycation in the diabetic subjects. A similar increase in permeability was observed in type 1 diabetic subjects on continuous subcutaneous insulin infusion, although it was not related to glycaemic control 

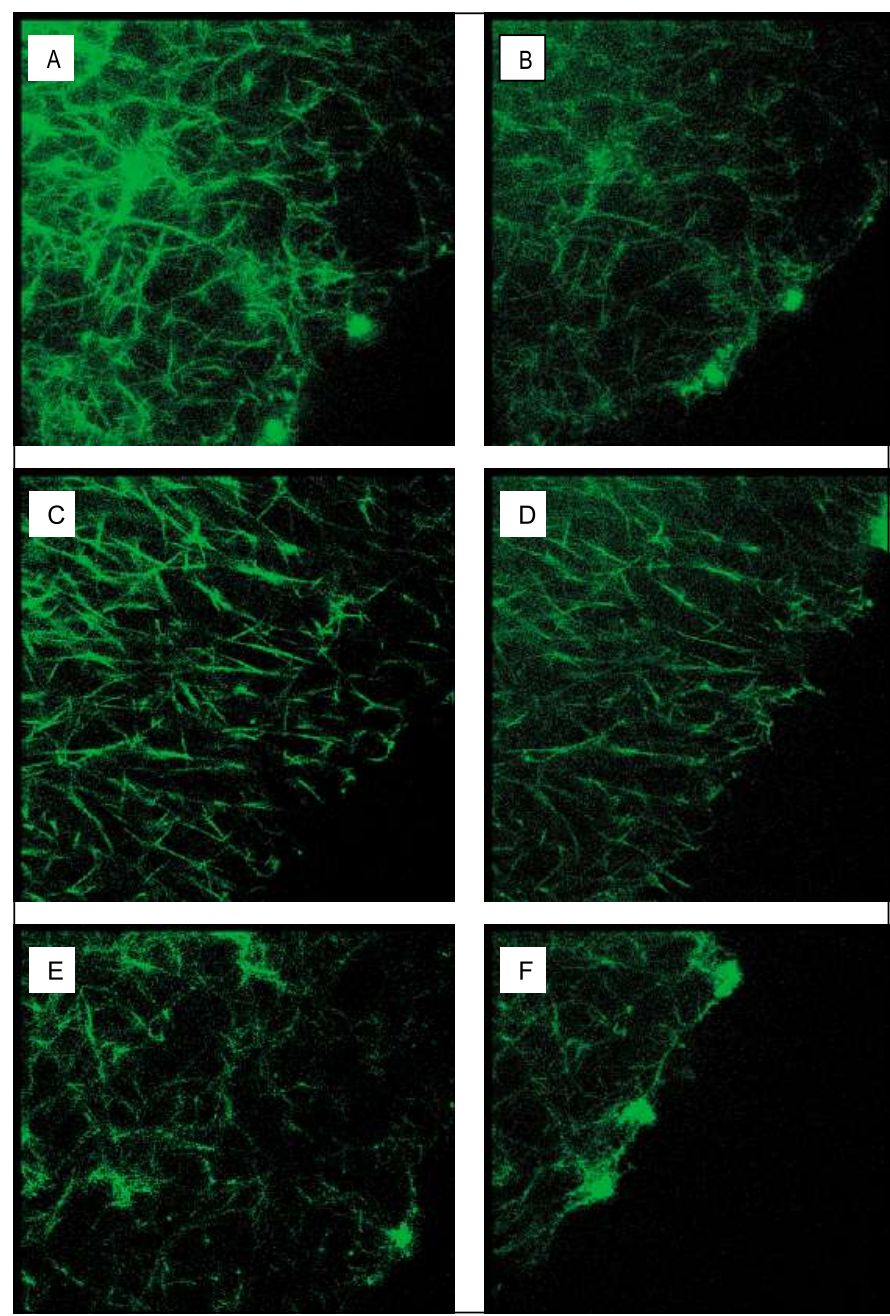

Figure 3: Representative 3D reconstructed images of purified fibrin clots from uncontrolled diabetic subjects $(A, B)$, controlled diabetic subjects (C, D) and non-diabetic subjects (E, F) obtained by confocal microscopy $(107 \times 107 \times 16 \mu \mathrm{m})$. Lysis was induced by the addition of $\mathrm{I} \mu \mathrm{g} / \mathrm{ml}$ tPA to the lysis front. Lysis times illustrated are after $0(A, C, E)$ and 10 min $(B, D, E)$.

(16). The diabetic subjects also displayed a significantly faster rate of lateral fibrin aggregation (slope) and a slower lysis rate than the non-diabetic subjects, which decreased and increased, respectively, with glycaemic control and reduced levels of fibrinogen glycation. These changes in slope and lysis rate, although small in comparison to the differences between diabetic and nondiabetic subjects, were consistently seen in all of the selected diabetic patients in the sub-sample. Permeability, slope and lysis rate correlated significantly with markers of glycaemic control such as $\mathrm{HbAl}$ c, fasting venous glucose and fibrinogen glycation, confirming the observed changes were related to changes observed in glucose concentrations. This may explain why the decrease in the rate of lateral association reached significance in the subgroup, but not in the total diabetic group, as the decrease in fibrinogen glycation in the total group was on average smaller than that of the subgroup and may therefore have diluted the decrease in the slope in the total group.

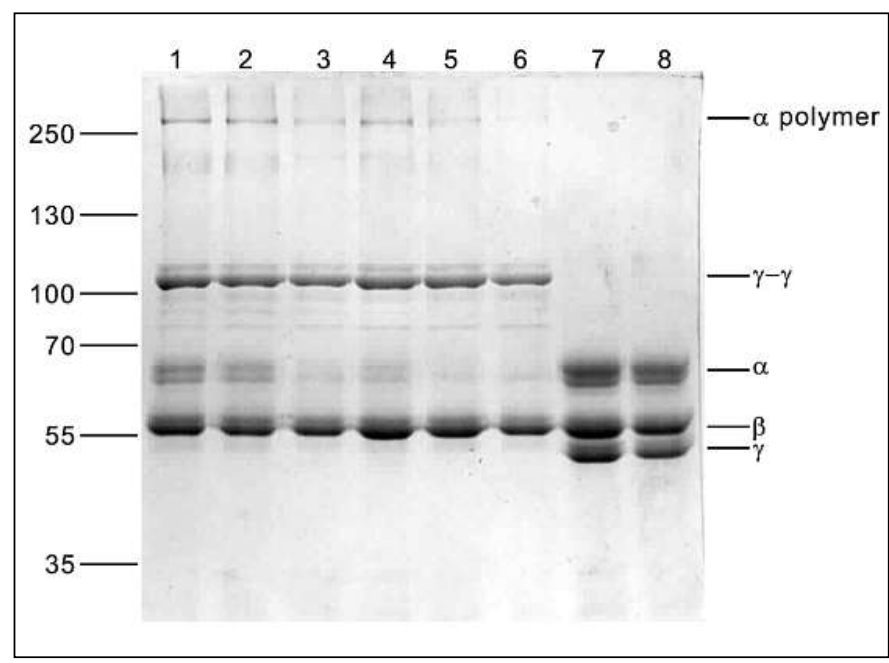

Figure 4: SDS-PAGE of FXIIla crosslinking of fibrin clots, showing the effect of glycaemic control. Tracks I, 3 and 5 are fibrin from uncontrolled diabetic patients after incubation with FXIIla for 10, 45 and $90 \mathrm{~min}$, respectively. Tracks 2, 4 and 6 are fibrin from controlled diabetic patients after the same incubation times. Tracks 7 and 8 are samples from uncontrolled and controlled diabetic patients respectively after 90 min of incubation without FXIII.

The increase in permeability upon achievement of glycaemic control reflects an increased pore size of the fibrin network and may be a result of altered clot formation kinetics. The decrease in the slope of the turbidity curve upon glycaemic control indicates that the kinetics of polymerization, specifically lateral aggregation, are modified as a result of the decrease in glycation, resulting in an increased pore size. The rate of lateral aggregation may be influenced by factors such as modulation of binding affinity or possible conformational and charge changes (29), as has been documented for calcium $(30)$ and chloride $(31,32)$. Similar changes may be induced by the presence of glucose on the fibrinogen molecule. Despite the increased permeability observed, there was, however, no significant difference in median fiber diameter between the uncontrolled and controlled diabetic subjects. This may be explained by the fact that the permeability measurement is a functional measurement which is also a more sensitive method than electron microscopy and is therefore able to detect smaller differences. In addition, the diabetic condition is complex, with various factors having varying degrees of influence on the different aspects of fibrin network structure, which may lead to the seemingly inconsistent results. Similar discrepancies were observed by the group of Dunn et al. (10) who found clots from diabetic patients to be more dense and less porous than that of control patients, but having increased, rather than decreased, fiber diameters.

Although this study showed no change in the mechanical properties of the clots after achievement of glycaemic control, the diabetic subjects in comparison with non-diabetic subjects, had clots with a lower proportion of the inelastic to elastic component of deformation both before and after achievement of glycaemic control. These mechanical properties of fibrin are functionally essential. Its structure has to be be strong enough to withstand the pressure of arterial blood flow and these properties 
will determine whether a thrombus will deform reversibly or irreversibly, rupture or embolize, under the pressure of flowing blood (33).

Diabetes is considered a hypercoagulable state due to, amongst other reasons, a relatively inhibited fibrinolytic system (34). Ex-vivo lysis rates of type 2 diabetic subjects have been shown to be decreased when compared to non-diabetic subjects (15), using tPA as a lytic agent. Our results are in agreement with these findings. We demonstrated for the first time that lysis rate increases in type 2 diabetic subjects when glycaemic control is achieved. While lysis rate, permeability and the rate of lateral aggregation correlated significantly with markers of glycaemic control, glycation of fibrinogen may not be the only cause for the observed changes. In addition to hyperglycaemia, poor glycaemic control is also associated with oxidative and carbonyl stress (35), which in turn have been shown to alter the structure of the fibrinogen molecule (36). It is important to note that the differences in lysis rates observed in this study were obtained using non-crosslinked clots and the differences in lysis rates can therefore not be attributed to possible differences in FXIIIa-induced crosslinking. Dunn et al. (15) demonstrated that binding of both tPA and plasminogen to fibrin is impaired in diabetic subjects and that there is a corresponding decrease in plasmin generation on the clot surface, which in part explains the reduced lysis rates of diabetic subjects. These changes in enzyme kinetics may be directly related to the binding of additional molecules to fibrin as a result of hyperglycaemia or indirectly through the resultant altered fibrin network architecture (e.g. increased pore size and higher proportion of thicker fibers), which has been shown to be a modulator of fibrinolysis speed $(3,4)$, or most likely a combination of both.

The effect of fibrinogen glycation on FXIIIa-induced crosslinking is not clear. No differences in either $\gamma$ - or $\alpha$-chain crosslinking were observed when clots made from in vitro glycated and normal fibrinogen were compared (6-8). When diabetic and non-diabetic subjects were compared, $\alpha$-chain crosslinking of diabetic subjects were previously found to be either decreased (12) or increased (10), and in our study, comparable to non-diabetic subjects. These discrepancies may be attributed to the different sample populations studied, differences in study designs and therefore different levels of fibrinogen glycation, or glycation of FXIII itself, or differences in analytical procedures. In our study there was additionally no discernable differences in FXIIIa-induced crosslinking observed between uncontrolled and controlled diabetic subjects.

In conclusion, using a purified model, we determined that achievement of glycaemic control alters the kinetics of fibrin polymerization, specifically lateral aggregation and improves permeability and lysis rate which may aid in the alleviation of the hypercoagulable state of diabetic subjects. Despite the fact that these variables were improved in all subjects, the changes observed in lateral aggregation and lysis rate were relatively small compared to the initial differences observed between the uncontrolled diabetic and non-diabetic subjects. This observation confirms that diabetes is a complex condition with many metabolic derangements having modulating effects on fibrin network structure, such as fibrinogen concentration, as was demonstrated in the plasma model (14). Therefore, the effect of individual contributing factors on fibrin network characteristics and the relative magnitude of the consequences amongst the many other causal factors in vivo, where whole blood clots are formed in the presence of platelets and blood flow, remain to be elucidated.

\section{Acknowledgements}

We appreciate the advice and help of Dr. Rustem I. Litvinov and Irina Chernysh, especially with the viscoelastic measurements. The study was funded by research funds from the North-West University, the University of Pretoria, Sugar Association (Project 209) and NIH grant HL30954. Insulin was provided by Sanofi-Aventis and Novo-Nordisk and glucometers by Roche Diagnostics.

\section{References}

1. Rett $\mathrm{K}$. The relation between insulin resistance and cardiovascular complications of the insulin resistance syndrome. Diabetes Obes Metab 1999; 1 (Suppl 1): S8-16.

2. Fatah K, Silveira A, Tornvall P, et al. Proneness to formation of tight and rigid fibrin gel structures in men with myocardial infarction at a young age. Thromb Haemost 1996; 76: 535-540.

3. Collet JP, Allali Y, Lesty C, et al. Altered fibrin architecture is associated with hypofibrinolysis and premature coronary atherothrombosis. Arterioscler Thromb Vasc Biol 2006; 26: 2567-2573.

4. Collet JP, Park D, Lesty C, et al. Influence of fibrin network conformation and fibrin fiber diameter on fibrinolysis speed: dynamic and structural approaches by confocal microscopy. Arterioscler Thromb Vasc Biol 2000; 20: 1354-1361

5. Bobbink IW, Tekelenburg WL, Sixma JJ, et al. Glycated proteins modulate tissue-plasminogen activatorcatalyzed plasminogen activation. Biochem Biophys Res Commun 1997; 240: 595-601.

6. Krantz S, Lober M, Thiele M, et al. Properties of in vitro nonenzymatically glycated plasma fibrinogens. Exp Clin Endocrinol 1987; 90: 37-45.

7. Ney KA, Pasqua JJ, Colley KJ, et al. In vitro preparation of nonenzymatically glucosylated human trans- ferrin, alpha 2-macroglobulin, and fibrinogen with preservation of function. Diabetes 1985; 34: 462-470. 8. Mirshahi M, Soria J, Soria C, et al. Glycosylation of human fibrinogen and fibrin in vitro. Its consequences on the properties of fibrin(ogen). Thromb Res 1987; 48: 279-289.

9. Brownlee M, Vlassara H, Cerami A. Nonenzymatic glycosylation reduces the susceptibility of fibrin to degradation by plasmin. Diabetes 1983; 32: 680-684. 10. Dunn EJ, Ariens RA, Grant PJ. The influence of type 2 diabetes on fibrin structure and function. Diabetologia 2005; 48: 1198-1206.

11. Nair CH, Azhar A, Wilson JD, et al. Studies on fibrin network structure in human plasma. Part II--Clinical application: diabetes and antidiabetic drugs. Thromb Res 1991; 64: 477-485.

12. Lutjens A, Jonkhoff-Slok TW, Sandkuijl C, et al Polymerisation and crosslinking of fibrin monomers in diabetes mellitus. Diabetologia 1988; 31: 825-830.

13. Jorneskog G, Egberg N, Fagrell B, et al. Altered properties of the fibrin gel structure in patients with IDDM. Diabetologia 1996; 39: 1519-1523.

14. Pieters M, Covic N, Loots dT, et al. The effect of glycaemic control on fibrin network structure of type 2 diabetic subjects. Thromb Haemost 2006; 96: 623-629.
15. Dunn EJ, Philippou H, Ariens RA, et al. Molecular mechanisms involved in the resistance of fibrin to clot lysis by plasmin in subjects with type 2 diabetes mellitus. Diabetologia 2006; 49: 1071-1080.

16. Jorneskog G, Hansson LO, Wallen NH, et al. Increased plasma fibrin gel porosity in patients with Type I diabetes during continuous subcutaneous insulin infusion. J Thromb Haemost 2003; 1: 1195-1201.

17. Dunn EJ, Ariens RA. Fibrinogen and fibrin clot structure in diabetes. Herz 2004; 29: 470-479.

18. Barazzoni R, Zanetti M, Davanzo G, et al. Increased fibrinogen production in type 2 diabetic patients without detectable vascular complications: correlation with plasma glucagon concentrations. J Clin Endocrinol Metab 2000; 85: 3121-3125.

19. Pieters M, van Zyl DG, Rheeder P, et al. Glycation of fibrinogen in uncontrolled diabetic patients and the effects of glycaemic control on fibrinogen glycation. Thromb Res 2007; 120: 439-446.

20. Takebe M, Soe G, Kohno I, et al. Calcium ion-dependent monoclonal antibody against human fibrinogen: preparation, characterization, and application to fibrinogen purification. Thromb Haemost 1995; 73: 662-667.

21. Blomback B, Carlsson K, Fatah K, et al. Fibrin in human plasma: gel architectures governed by rate and 
nature of fibrinogen activation. Thromb Res 1994; 75: $521-538$

22. Fatah K, Hamsten A, Blomback B, et al. Fibrin gel network characteristics and coronary heart disease: relations to plasma fibrinogen concentration, acute phase protein, serum lipoproteins and coronary atherosclerosis. Thromb Haemost 1992; 68: 130-135.

23. Langer BG, Weisel JW, Dinauer PA, et al. Deglycosylation of fibrinogen accelerates polymerization and increases lateral aggregation of fibrin fibers. J Biol Chem 1988; 263: 15056-15063.

24. Woodhead JL, Nagaswami C, Matsuda M, et al. The ultrastructure of fibrinogen Caracas II molecules, fibers, and clots. J Biol Chem 1996; 271: 4946-4953. 25. Sakharov DV, Nagelkerke JF, Rijken DC. Rearrangements of the fibrin network and spatial distribution of fibrinolytic components during plasma clot lysis. Study with confocal microscopy. J Biol Chem 1996; 271: 2133-2138.
26. Laemmli UK. Cleavage of structural proteins during the assembly of the head of bacteriophage T4. Nature 1970; 227: 680-685.

27. Field AP. Discovering statistics using SPSS. 2nd ed. London: Sage Publications; 2005.

28. Ryan EA, Mockros LF, Weisel JW, et al. Structural origins of fibrin clot rheology. Biophys J 1999; 77 : 2813-2826

29. Weisel JW, Veklich Y, Gorkun O. The sequence of cleavage of fibrinopeptides from fibrinogen is important for protofibril formation and enhancement of lateral aggregation in fibrin clots. J Mol Biol 1993; 232: 285-297.

30. Mihalyi E. Clotting of bovine fibrinogen. Kinetic analysis of the release of fibrinopeptides by thrombin and of the calcium uptake upon clotting at high fibrinogen concentrations. Biochemistry 1988; 27: 976-982. 31. Di Stasio E, Nagaswami C, Weisel JW, et al. Cl- regulates the structure of the fibrin clot. Biophys J 1998; 75: 1973-1979.

32. Veklich YI, Gorkun OV, Medved LV, et al. Carboxyl-terminal portions of the alpha chains of fibrinogen and fibrin. Localization by electron microscopy and the effects of isolated alpha $\mathrm{C}$ fragments on polymerization. J Biol Chem 1993; 268: 13577-13585.

33. Weisel JW. The mechanical properties of fibrin for basic scientists and clinicians. Biophys Chem 2004; 112: 267-276.

34. Carr ME. Diabetes mellitus: a hypercoagulable state. J Diabetes Complications 2001; 15: 44-54.

35. Baynes JW, Thorpe SR. Role of oxidative stress in diabetic complications: a new perspective on an old paradigm. Diabetes 1999; 48: 1-9.

36. Shacter E, Williams JA, Levine RL. Oxidative modification of fibrinogen inhibits thrombin-catalyzed clot formation. Free Radic Biol Med 1995; 18: 815-821. 